\title{
The role of chemotherapy in the management of fungal endocarditis following homograft valve replacement
}

\author{
WILLIAM WAIN* \\ $\mathrm{Ph}$. D. \\ MOHAMED AHMED \\ M.R.C.P. \\ RICHARD THOMPSON \\ M.R.C.P. \\ Magdi Yacoub \\ F.R.C.S. \\ * National Heart Hospital, London, and Harefield Hospital, Middlesex
}

\begin{abstract}
Summary
Initial intensive combined chemotherapy, with late re-operation when indicated, appears to give better results than immediate excision of the infected valve. The combination chemotherapy used in this series was amphotericin $B$ (up to $100 \mathrm{mg} /$ patient/day) and flucytosine (up to $12 \mathrm{~g} /$ patient/day). Serious side effects attributed to this chemotherapy were extremely rare. Candida infections were the most common and the Aspergillus infections were uniformly fatal. Embolectomies and excisions of large mycotic aneurysms were generally successful. Re-operation for late valve malfunction was common and yielded satisfactory results.
\end{abstract}

\section{Introduction}

Cardiac valve replacement is associated with a higher susceptibility to infective endocarditis (Wilson, 1977) and fungal endocarditis is particularly dangerous because of the problem of antifungal chemotherapy (McLeod and Remington, 1977). Antibiotic sterilized homograft valves have been used since 1968 (Stinson et al., 1968; Barratt-Boyes and Roche, 1969; Yacoub and Kittle, 1970; Lockey et al., 1972) and are considered by the authors to be one of the best available valve substitutes. Fungal endocarditis following homograft valve replacement remains an important cause of mortality and morbidity. The purpose of this paper is to attempt to define the clinical course and results of different methods of treatment.

\section{Patients and methods}

Homograft valves were inserted (by M.Y.) in 1207 patients between 1969 and 1978, 558 in the aortic position, 528 in the mitral position and 121 patients received replacement of both aortic and mitral valves. Concomitant surgery included closure of septal defects, valvotomy, and coronary artery bypass grafts with autologous saphenous vein. No other grafts or prostheses were used in these patients.

\section{Valve preparation}

Homograft valves were collected from routine post-mortem material within $48 \mathrm{hr}$ of death and treated in the antibiotic mixture of Yacoub, Knight and Towers (1973). This contained carbenicillin $10 \mathrm{~g} / \mathrm{l}$, cephaloridine $10 \mathrm{~g} / \mathrm{l}$, neomycin $5 \mathrm{~g} / \mathrm{l}$, polymixin $B \quad 0.7 \mathrm{~g} / 1$ and amphotericin $\mathrm{B} 0.25 \mathrm{~g} / 1$ in Hanks balanced salt solution. After $24 \mathrm{hr}$ at $4^{\circ} \mathrm{C}$ the valves were transferred to a tissue culture medium (199) containing $7 \%$ serum and nystatin $0.5 \mathrm{~g} / 1$ and stored at $4^{\circ} \mathrm{C}$ until used. Samples of the valves were taken at the transfer stage and used for microbiological screening. The valves were used as soon as possible after treatment, preferably within 7 days and never used after 8 weeks. Further samples of the valve were taken for screening at the time of implantation. Another 96 valves were treated in solution B of Lockey et al. (1972), 30 with solution C of Waterworth et al. (1974) and 49 with the DANYNM solution of Wain et al. (1977).

\section{Results}

Between 1969 and 1978, 27 patients developed fungal endocarditis, an overall incidence of $2 \cdot 2 \%$ for the 1207 patients, with a value of $4.9 \%$ in the aortic and mitral double valve replacements, $2.1 \%$ in the aortic valves and $1.7 \%$ in the mitral valve replacements.

\section{Infecting fungi and time of onset of endocarditis}

Candida spp. were the most common isolates $(85 \%)$ and $C$. albicans the most frequent $(70 \%)$. Three of the non-Candida fungi were Aspergillus spp. and there was one isolate of Petriellidium boydii (Allescheria boydii). These 27 fungal isolates are shown in Fig. 1 together with the time of onset of endocarditis related to the date of insertion of the homograft valve. The 3 Aspergillus spp. occurred later than the majority of Candida infections, although C. parakrusei was isolated from 2 cases at 30 and 36 months after the original operation. More than half $(55 \%)$ 


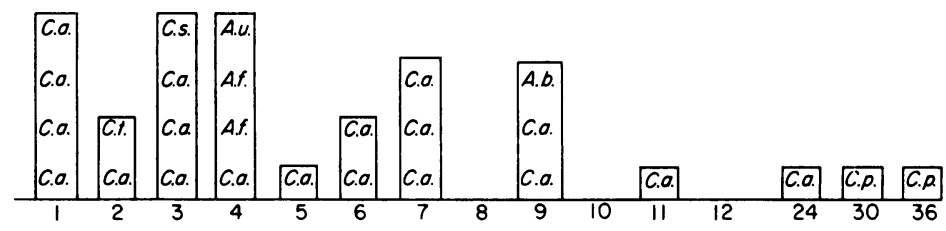

FIG. 1. Onset of endocarditis, and fungal species; months between homograft insertion and onset of endocarditis. A.b. = Allescheria boydii. A.f. $=$ Aspergillus fumigatus. A.u. =Aspergillus ustus. C.a=Candida albicans. C.p.=Candida parakrusei. C.s. $=$ Candida stellatoidea. C.t. $=$ Candida tropicalis.

of the infections occurred within 5 months of the homograft valve insertion.

\section{Clinical presentation}

Six patients presented with severe haemodynamic disturbance and required an immediate re-operation to replace the damaged valve. Another 12 patients presented with major emboli, 6 of which required embolectomy. Five more patients presented with minor emboli, such as clubbed fingers, petechial rashes or Ossler's nodes. A further 4 patients had pyrexia as their principal clinical manifestation.

\section{Fungal cultures}

Positive cultures were obtained for all 27 patients in association with the clinical manifestation of fungal endocarditis. Nineteen had positive blood cultures $(70 \%), 10$ grew fungus from valvar vegetations, removed at operation or post mortem $(37 \%)$, 4 had positive cultures from samples removed at embolectomy and 2 had positive cultures from the screening samples of the homograft. None of the 4 patients with Aspergillus or Petriellidium had positive blood cultures at the time of diagnosis. Only one positive blood culture for $A$. fumigatus was recorded at any time during the infections. Four of the patients with Candida infection had negative blood cultures and in these patients positive cultures were obtained from emboli (2) and valvar vegetations (2).

\section{Chemotherapy}

Six of the patients died within 3 days of diagnosis of endocarditis before any effective chemotherapy could be initiated. The remaining 21 patients were treated with intensive chemotherapy of amphotericin B in combination with flucytosine. Flucytosine was not used in 2 cases in which the organism was known to be resistant to the drug. Amphotericin B (i.v.) was given in increasing doses up to $100 \mathrm{mg} /$ day (55 000 i.u.) usually for 6 weeks. One patient was treated for 9 weeks and several had shorter treatment, interrupted treatment or reduced doses of amphotericin B dictated by the patient's response to the drug. Flucytosine was given orally at doses of up to $12 \mathrm{~g}$ / day, usually for 3 months. One patient received flucytosine for more than 6 months without adverse effect. The dose was altered with due regard to serum levels and occasionally administered intravenously in unconscious patients, or those with problems of nausea.

One patient was treated with flucytosine only because of an initial adverse reaction to amphotericin B. Clotrimazole and miconazole were used sequentially with the combined amphotericin B and flucytosine chemotherapy in 2 patients in which the fungus was not responding. In neither case were these drugs any more effective than the normal combination.

\section{Immediate re-operation}

Five patients presented with severe haemodynamice disturbance which required an immediate re- 8 operation to replace the damaged valve. These included the 3 patients with Aspergillus and the $P$. boydii. Two of these patients died within 2 weeks from an overwhelming infection unresponsive to the subsequent chemotherapy. Two more patients succumbed to a recurrence of the infection more than 3 months after the immediate re-operation. Only one patient survived for more than 4 months and in that case there was a recurrence of the infection and re-operation was followed by an overwhelming infection which did not respond to chemotherapy.

\section{Intensive initial chemotherapy}

Sixteen patients were treated with intensive initial chemotherapy and 9 of these did not undergo subsequent re-operation. Three of these died within 2 weeks of diagnosis, owing to uncontrolled infection. Two more died within 6 weeks with some indication of drug toxicity and fungal toxaemia. One patient refused re-operation and died after 11 months and 3 patients are alive, 15 to 27 months after the start of chemotherapy. It is anticipated that these 3 patients will eventually require a re-operation to replace the damaged valve

Seven of the 16 patients who received intensive initial chemotherapy have had a re-operation to replace the valve. The operation was necessary 
because of the patient's cardiac status and was performed 2 weeks after initiation of chemotherapy in one case and after 40 months in another. One of these patients died, with a low cardiac output, within 5 days of the re-operation at 10 months and the remaining 6 are alive 33 to 87 months after endocarditis, an average survival of 50 months. Nine of these 16 patients, treated with intensive initial chemotherapy are alive 15 to 87 months after the onset of the infection, an average survival of $\mathbf{4 0}$ months without evidence of recurrence.

\section{Complications of chemotherapy}

All 18 patients treated with chemotherapy for more than 2 weeks, including 3 patients who underwent re-operation, experienced some temporary renal disturbance. Sometimes this was sufficiently severe to modify the dose of amphotericin B therapy. Seven of these 18 patients experienced some gastrointestinal disturbance and 4 succumbed to a recurrence of their uncontrolled infection. One patient developed agranulocytosis whilst on flucytosine, but this was thought to be secondary to acute streptococcal septicaemia (superinfection) and was successfully treated with antibiotics and blood transfusion. This patient was then treated with flucytosine for a further 5 months with a maintaining leucocyte count in excess of $5000 / \mathrm{mm}^{3}$. There was one patient who developed jaundice with temporary hepatic disturbance and one patient who attempted suicide.

\section{Complications of infection}

Other complications associated with the endocarditis included 12 emboli, 8 of which were removed and 3 mycotic aneurysms, 2 of which were excised and a section of the affected artery replaced with autogenous saphenous vein. Seven of the 8 patients who underwent embolectomy have survived for 17 to 87 months as have the 2 patients from whom the aneurysms were excised. Two other patients developed terminal gangrene with multiple peripheral emboli.

\section{Serology}

Initial agglutination titres to $C$. albicans varied from $1 / 21$ to $1 / 512$ and generally fell to $1 / 20$ to $1 / 64$ by 4 months. Two of the patients who died during chemotherapy showed a rise in titre $(1 / 160$ to $1 / 320$; $1 / 64$ to $1 / 128$ ) but generally a sustained fall in the agglutination titre was used to support the decision to stop amphotericin B therapy at 6 weeks.

\section{Discussion}

Nine $(33 \%)$ patients who have not shown any sign of recurrence between 17 and 87 months after treatment of fungal endocarditis were considered as examples of successful chemotherapy. The deaths of $11(41 \%)$ of the patients within 2 weeks of the diagnosis of fungal endocarditis probably represents uncontrolled fulminating infection. The inability to control the infection over several months in a further 3 patients who had required an immediate reoperation for severe haemodynamic reasons is seen as support for the policy of intensive initial chemotherapy. This policy is based on the belief that the trauma of cardiopulmonary bypass surgery imposes an unacceptable strain on such acutely ill patients. It is believed that an immediate re-operation affects the patient's defence system and permits a subsequent flare-up of the systemic infection which cannot adequately be controlled by chemotherapy. In contrast, intensive initial chemotherapy, to control the infection, can permit the patient to recover from the acute symptoms of endocarditis. The cardiac status of the patient may then be assessed and a decision made about the eventual replacement of the affected valve. The effectiveness of this policy is supported by the fact that $9(69 \%)$ of the 16 patients who received intensive initial chemotherapy for more than 2 weeks have a long-term survival of 17-87 months.

\section{References}

Barratt-Boyes, B.G. \& Roche, A.M.G. (1969) A review of aortic valve homografts over a six and one half year period. Annals of Surgery, 170, 483.

Lockey, E., Al-Janabi, N., Gonzalez-Lavin, L. \& Ross, D.N. (1972) A method of sterilizing and preserving fresh allograft heart valves. Thorax, 27, 398.

McLeod, R. \& Remington, J.S. (1977) Postoperative fungal endocarditis. In: Infections of Prosthetic Heart Valves and Vascular Grafts (Ed. by Duma, R.J.). University Park Press, Baltimore.

Stinson, E.B., Angell, W.W., Iben, A.B. \& Shumway, N.E. (1968) Aortic valve replacement with the fresh valve homograft. American Journal of Surgery, 116, 204.

Wain, W.H., Pearce, H.M., Riddell, R.W. \& Ross, D.N. (1977) A re-evaluation of the antibiotic sterilisation of heart valve allografts. Thorax, 32, 740.

Waterworth, P.M., Lockey, E., Berry, E.M. \& Pearce, H.M. (1974) A critical investigation into the antibiotic sterilization of heart valve homografts. Thorax, 29, 432.

WILson, W.R. (1977) Prosthetic valve endocarditis: incidence, anatomic location, cause, morbidity and mortality. In: Infections of Prosthetic Heart Valves and Vascular Grafts (Ed. by Duma, R.J.). University Park Press, Baltimore.

YACoub, M.H. \& Kittle, F. (1970) Sterilization of valve homografts by antibiotic solutions. Circulation, $41 / 42$ (Suppl. 2), 29.

Yacoub, M.H., KNight, E.J. \& Towers, M. (1973) Proceedings: Aortic valve replacement using fresh unstented homogsraft. Thoraxchirurgie, 21, 451. 